\title{
Curve fitting predicting the number of daily deaths due to the COVID-19 in the near future
}

yoshiyasu takefuji ${ }^{1}$

${ }^{1}$ Affiliation not available

November 18, 2020

I agree with their implications on the weakness of the US healthcare system ${ }^{1,2}$. As of Oct. 30 in 2020 using the last 200 days open data ${ }^{3}$, the number of daily deaths due to the COVID-19 in the US can be predicted by the state-of-the-art curve fitting with the $6^{\text {th }}$ degree polynomial:

Nov. 6 daily deaths 959 ,

Nov. 13 daily deaths 1017, and

Nov. 20 daily deaths 1002 .

Fig. 1 shows the predicted three red points on the number of deaths on Nov. 6, Nov. 13 and Nov. 21 respectively. The graph was created by the curve-fitting Python program: usdaily.py ${ }^{4}$. If the US does not change the current policy against the COVID-19 pandemic, the number of daily deaths will not be alleviated in the next three weeks.

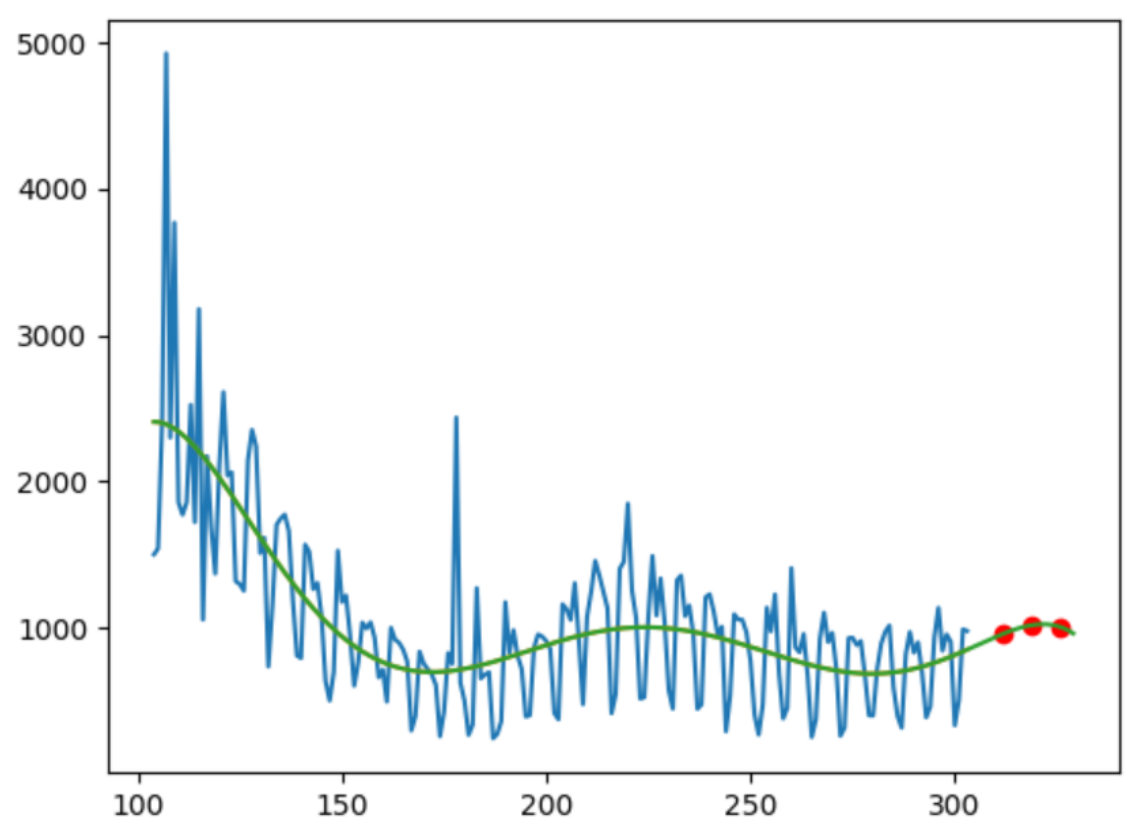

Fig. 1 predicted number of deaths on Nov.6, 13 and 21 


\section{References:}

1. October 29, 2020 N Engl J Med 2020; 383:e105 DOI: 10.1056/NEJMsr2031592

2. October 21, 2020 DOI: 10.1056/NEJMp2028209

3.https://covid.ourworldindata.org/data/ecdc/new_deaths.csv

4. https://github.com/ytakefuji/US 